\title{
New Weighted Norm Inequalities for Pseudodifferential Operators and Their Commutators
}

\author{
The Anh Bui ${ }^{1,2}$ \\ ${ }^{1}$ Department of Mathematics, Ho Chi Minh City University of Pedagogy, Ho Chi Minh City 70000, Vietnam \\ 2 Department of Mathematics, Macquarie University, Sydney, NSW 2109, Australia
}

Correspondence should be addressed to The Anh Bui; bt_anh80@yahoo.com

Received 18 August 2012; Accepted 13 November 2012

Academic Editor: Serge Nicaise

Copyright (C) 2013 The Anh Bui. This is an open access article distributed under the Creative Commons Attribution License, which permits unrestricted use, distribution, and reproduction in any medium, provided the original work is properly cited.

This paper is dedicated to study weighted $L^{p}$ inequalities for pseudodifferential operators with amplitudes and their commutators by using the new class of weights $A_{p}^{\infty}$ and the new $\mathrm{BMO}$ function space $\mathrm{BMO}_{\infty}$ which are larger than the Muckenhoupt class of weights $A_{p}$ and classical BMO space BMO, respectively. The obtained results therefore improve substantially some well-known results.

\section{Introduction and the Main Results}

For $f \in C_{0}^{\infty}\left(\mathbb{R}^{n}\right)$ a pseudodifferential operator given formally by

$$
T_{a} f(x)=\frac{1}{(2 \pi)^{n}} \iint_{\mathbb{R}^{n}} a(x, y, \xi) e^{i\langle x-y, \xi\rangle} f(y) d y d \xi
$$

where the amplitude $a$ satisfies certain growth conditions. The boundedness of pseudodifferential operators has been studied extensively by many mathematicians; see, for example, [1-7] and the references therein. One of the most interesting problems is studying the weighted norm inequalities for pseudodifferential operators and their commutators with BMO function; see, for example, [5-9].

In this paper we consider the following classes of symbols and amplitudes $a$ (in what follows we set $\langle x\rangle=\left(1+|x|^{2}\right)^{1 / 2}$ ).

Definition 1. Let $a: \mathbb{R}^{n} \times \mathbb{R}^{n} \times \mathbb{R}^{n} \rightarrow \mathbb{R}^{n}$ and $m \in \mathbb{R}$, $\rho \in[0,1]$ and $\delta \in[0,1]$.

(a) We say $a \in A_{\rho, \delta}^{m}$ when for each triple of multi-indices $\alpha, \beta$, and $\gamma$ there exists a constant $C$ such that

$$
\left|\partial_{\xi}^{\alpha} \partial_{x}^{\beta} \partial_{y}^{\gamma} a(x, y, \xi)\right| \leq C\langle\xi\rangle^{m-\rho|\alpha|+\delta|\beta+\gamma|} .
$$

(b) We say $a \in L^{\infty} A_{\rho, \delta}^{m}$ when for each triple of multiindices $\alpha, \beta$, and $\gamma$ there exists a constant $C$ such that

$$
\left\|\partial_{\xi}^{\alpha} \partial_{y}^{\beta} a(\cdot, y, \xi)\right\|_{L^{\infty}} \leq C\langle\xi\rangle^{m-\rho|\alpha|+\delta|\beta|} .
$$

Definition 2. Let $a: \mathbb{R}^{n} \times \mathbb{R}^{n} \rightarrow \mathbb{R}^{n}$ and $m \in \mathbb{R}, \rho \in[0,1]$ and $\delta \in[0,1]$.

(a) We say $a \in S_{\rho, \delta}^{m}$ when for each pair of multi-indices $\alpha$ and $\beta$ there exists a constant $C$ such that

$$
\left|\partial_{\xi}^{\alpha} \partial_{x}^{\beta} a(x, \xi)\right| \leq C\langle\xi\rangle^{m-\rho|\alpha|+\delta|\beta|} .
$$

(b) We say $a \in L^{\infty} S_{\rho}^{m}$ when for each multi-indices $\alpha$ there exists a constant $C$ such that

$$
\left\|\partial_{\xi}^{\alpha} a(\cdot, \xi)\right\|_{L^{\infty}} \leq C\langle\xi\rangle^{m-\rho|\alpha|} .
$$

It is easy to see that $S_{\rho, \delta}^{m} \subset A_{\rho, \delta}^{m}, L^{\infty} S_{\rho}^{m} \subset L^{\infty} A_{\rho, \delta}^{m}$, $S_{\rho, \delta}^{m} \subset L^{\infty} S_{\rho}^{m}$, and $A_{\rho, \delta}^{m} \subset L^{\infty} A_{\rho, \delta}^{m}$. The classes $A_{\rho, \delta}^{m}$ and $S_{\rho, \delta}^{m}$ were studied in $[3,8]$. For further information about these two classes, we refer the reader to, for example, $[3,10]$. The class $L^{\infty} S_{\rho}^{m}$ was introduced by [11], and it is the natural generalization of the class $S_{\rho, \delta}^{m}$. This class is much rougher than that considered in $[6,7]$. The amplitude class $L^{\infty} A_{\rho, \delta}^{m}$ 
in Definition 1 is rough in the $x$ variable, but smooth in the $y$ variable. This is smaller than the class $L^{\infty} A_{\rho}^{m}$ introduced in [5] but includes the class $A_{\rho, \delta}^{m}$.

The aim of this paper is to study the weighted norm inequalities for pseudodifferential operators $T_{a}$ and their commutators by using the new BMO functions and the new class of weights. Firstly, we would like to give brief definitions on the new class of weights and the new BMO function space (we refer to Section 2 for details).

The new classes of weights $A_{p}^{\infty}=\cup_{\theta>0} A_{p}^{\theta}$ for $p \geq 1$, where $A_{p}^{\theta}, \theta \geq 0$, is the set of those weights satisfying

$$
\left(\int_{B} w\right)^{1 / p}\left(\int_{B} w^{-1 /(p-1)}\right)^{1 / p^{\prime}} \leq C|B|\left(1+r_{B}\right)^{\theta}
$$

for all ball $B=B\left(x_{B}, r_{B}\right)$. We denote that $A_{\infty}^{\infty}=\cup_{p \geq 1} A_{p}^{\infty}$. It is easy to see that the new class $A_{p}^{\infty}$ is strictly larger than the Muckenhoupt class $A_{p}$. Indeed, for example, the weight $w(x)=1+|x|^{\gamma}$ with $\gamma>n(p-1)$ belongs to the class $A_{p}^{\infty}$, but it is not in $A_{p}$, for $p>1$, see, for example, [12].

The new $\mathrm{BMO}$ space $\mathrm{BMO}_{\theta}$ with $\theta \geq 0$ is defined as a set of all locally integrable functions $b$ satisfying

$$
\frac{1}{|B|} \int_{B}\left|b(y)-b_{B}\right| d y \leq C\left(1+r_{B}\right)^{\theta},
$$

where $B=B\left(x_{B}, r_{B}\right)$ and $b_{B}=(1 /|B|) \int_{B} b$. A norm for $b \in \mathrm{BMO}_{\theta}$, denoted by $\|b\|_{\theta}$, is given by the infimum of the constants satisfying (12). Clearly $\mathrm{BMO}_{\theta_{1}} \subset \mathrm{BMO}_{\theta_{2}}$ for $\theta_{1} \leq \theta_{2}$ and $\mathrm{BMO}_{0}=\mathrm{BMO}$. We define $\mathrm{BMO}_{\infty}=\cup_{\theta>0} \mathrm{BMO}_{\theta}$.

Our main result is the following theorem.

Theorem 3. Let $a \in L^{\infty} A_{\rho, \delta}^{m}$ with $m<n(\rho-1)$ or $a \in$ $L^{\infty} A_{1, \delta}^{0}, \delta \in[0,1]$. If $T_{a}$ is bounded on $L^{p}$ for all $1<p<\infty$, then

(a) $T_{a}$ is bounded on $L^{p}(w)$ for $1<p<\infty$ and $w \in A_{p}^{\infty}$;

(b) for any $b \in \mathrm{BMO}_{\infty}$, the commutator $\left[b, T_{a}\right]$ bounded on $L^{p}(w)$ for $1<p<\infty$ and $w \in A_{p}^{\infty}$.

In particular, the obtained results in (a) and (b) still hold for $w(x)=1+|x|^{\gamma}$ with $\gamma>n(p-1)$.

We would like to specify some applications of Theorem 3.

In [8], the author studied the weighted $L^{p}$ inequalities of $T_{a}$ when the symbol $a$ belongs to the class $S_{1, \delta}^{0} \subset L^{\infty} A_{1, \delta}^{0}$ with $\delta \in(0,1)$. It was proved that $T_{a}$ is bounded on $L^{p}(w)$ for $1<p<\infty, w \in A_{p}$. Recently, the author in [9] showed that $T_{a}$ and its commutator with a BMO function $\left[b, T_{a}\right]$ are bounded on $L^{p}(w)$ for $1<p<\infty$ and $w \in A_{p}^{\infty}$ by the different approach. Here, by using Theorem 3, we not only reobtain the boundedness of $T_{a}$ on $L^{p}(w)$ for $1<p<\infty$ and $w \in A_{p}^{\infty}$ but also obtain the new result on the boundedness of its commutator with $\mathrm{BMO}_{\infty}$ functions.

Corollary 4. Let $a \in S_{1, \delta}^{0} \subset L^{\infty} A_{1, \delta}^{0}, 0<\delta<1$. Then we have the following:

(i) $T_{a}$ is bounded on $L^{p}(w)$ for $1<p<\infty$ and $w \in A_{p}^{\infty}$; (ii) for each $b \in \mathrm{BMO}_{\infty}$, the commutator $\left[b, T_{a}\right]$ is bounded on $L^{p}(w)$ for $1<p<\infty$ and $w \in A_{p}^{\infty}$.

In particular, the obtained results in (i) and (ii) still hold for $w(x)=1+|x|^{\gamma}$ with $\gamma>n(p-1)$.

Now we consider the class $L^{\infty} S_{\rho}^{m}$. If $a \in L^{\infty} S_{\rho}^{m}$ with $\rho \in$ $[0,1]$ and $m<n(\rho-1)$, then the authors in [5] proved that the pseudodifferential operator $T_{a}$ and its commutators with BMO functions $\left[b, T_{a}\right]$ are bounded on $L^{p}(w)$ for $1<p<\infty$ and $w \in A_{p}$; see [5, Theorems 3.3 and 4.5]. So, Theorem 3 leads us to the following result.

Corollary 5. Let $a \in L^{\infty} S_{\rho}^{m}$ with $\rho \in[0,1]$ and $m<n(\rho-1)$. Then we have the following:

(i) $T_{a}$ is bounded on $L^{p}(w)$ for $1<p<\infty$ and $w \in A_{p}^{\infty}$;

(ii) for each $b \in B M O_{\infty}$, the commutator $\left[b, T_{a}\right]$ is bounded on $L^{p}(w)$ for $1<p<\infty$ and $w \in A_{p}^{\infty}$.

In particular, the obtained results in (i) and (ii) still hold for $w(x)=1+|x|^{\gamma}$ with $\gamma>n(p-1)$.

It was proved in [5, Theorem 3.7] that if $a \in L^{\infty} A_{\rho}^{m}$ with $0 \leq \rho \leq 1$ and $m<n(\rho-1)$, then $T_{a}$ and $\left[b, T_{a}\right]$ are bounded on $L^{p}(w)$ for $1<p<\infty$ and $w \in A_{p}$ with $b \in$ BMO. Therefore, in the light of Theorem 3 , we have the following:

Corollary 6. Let $a \in L^{\infty} A_{\rho, \delta}^{m}$ with $0 \leq \rho \leq 1$ and $m<n(\rho-1)$. Then we have the following:

(i) $T_{a}$ is bounded on $L^{p}(w)$ for $1<p<\infty$ and $w \in A_{p}^{\infty}$;

(ii) for each $b \in \mathrm{BMO}_{\infty}$, the commutator $\left[b, T_{a}\right]$ is bounded on $L^{p}(w)$ for $1<p<\infty$ and $w \in A_{p}^{\infty}$.

In particular, the obtained results in (i) and (ii) still hold for $w(x)=1+|x|^{\gamma}$ with $\gamma>n(p-1)$.

For smooth amplitudes, we have the following result.

Corollary 7. Let $a \in A_{\rho, \delta}^{n(\rho-1)}$ with $0<\rho \leq 1,0 \leq \delta<1$. Then we have the following:

(i) $T_{a}$ is bounded on $L^{p}(w)$ for $1<p<\infty$ and $w \in A_{p}^{\infty}$;

(ii) for each $b \in \mathrm{BMO}_{\infty}$, the commutator $\left[b, T_{a}\right]$ is bounded on $L^{p}(w)$ for $1<p<\infty$ and $w \in A_{p}^{\infty}$.

In particular, the obtained results in (i) and (ii) still hold for $w(x)=1+|x|^{\gamma}$ with $\gamma>n(p-1)$.

Proof. The remark in [1, page 11] tells us that $T_{a}$ is bounded on $L^{p}$ for $1<p<\infty$. Thanks to Theorem 3, we conclude that $T_{a}$ and $\left[b, T_{a}\right], b \in \mathrm{BMO}_{\infty}$ are bounded on $L^{p}(w)$ for $1<p<\infty$ and $w \in A_{p}^{\infty}$.

The outline of the paper is as follows. In Section 2, we first recall some definitions of the new class of weights $A_{p}^{\infty}$ and the new BMO function spaces $\mathrm{BMO}_{\infty}$. Then we also review some basic properties concerning $A_{p}^{\infty}$ and $\mathrm{BMO}_{\infty}$. Section 3 represents some kernel estimates for the pseudodifferential operator $T_{a}$. The proof of the main result will be given in Section 4. 


\section{Preliminaries}

To simplify notation, we will often just use $B$ for $B\left(x_{B}, r_{B}\right)$ and $|E|$ for the measure of $E$ for any measurable subset $E \subset$ $\mathbb{R}^{n}$. Also given $\lambda>0$, we will write $\lambda B$ for the $\lambda$-dilated ball, which is the ball with the same center as $B$ and with radius $r_{\lambda B}=\lambda r_{B}$. For each ball $B \subset \mathbb{R}^{n}$ we set that

$$
S_{0}(B)=B, \quad S_{j}(B)=2^{j} B \backslash 2^{j-1} B \quad \text { for } j \in \mathbb{N} .
$$

2.1. The New Class of Weights and New BMO Function Spaces. Recently, in [12], a new class of weights associated to Schrödinger operators $L:=-\Delta+V$, where the potential $V \in R H_{n / 2}$, the reverse Hölder class has been introduced. According to [12], the authors defined the new classes of weights $A_{p}^{L}=\cup_{\theta \geq 0} A_{p}^{L, \theta}$ for $p \geq 1$, where $A_{p}^{L, \theta}, \theta \geq 0$, is the set of those weights satisfying

$$
\left(\int_{B} w\right)^{1 / p}\left(\int_{B} w^{-1 /(p-1)}\right)^{1 / p^{\prime}} \leq C|B|\left(1+\frac{r}{\rho(x)}\right)^{\theta}
$$

for all ball $B=B(x, r)$. We denote that $A_{\infty}^{L}=\cup_{p \geq 1} A_{p}^{L}$, where the critical radius function $\rho(\cdot)$ is defined by

$$
\rho(x)=\sup \left\{r>0: \frac{1}{r^{n-2}} \int_{B(x, r)} V \leq 1\right\}, \quad x \in \mathbb{R}^{n} .
$$

In this paper, we consider the particular case when $\rho(\cdot) \equiv$ 1. In this situation the new classes of weights are defined by $A_{p}^{\infty}=\cup_{\theta \geq 0} A_{p}^{\theta}$ for $p \geq 1$, where $A_{p}^{\theta}, \theta \geq 0$ is the set of those weights satisfying

$$
\left(\int_{B} w\right)^{1 / p}\left(\int_{B} w^{-1 /(p-1)}\right)^{1 / p^{\prime}} \leq C|B|\left(1+r_{B}\right)^{\theta}
$$

for all ball $B=B\left(x_{B}, r_{B}\right)$. We denote that $A_{\infty}^{\infty}=\cup_{p \geq 1} A_{p}^{\infty}$.

It is easy to see that the new class $A_{p}^{\infty}$ is larger than the Muckenhoupt class $A_{p}$. The following properties hold for the new classes $A_{p}^{\infty}$; see [12, Proposition 5].

Proposition 8. The following statements hold:

(i) $A_{p}^{\infty} \subset A_{q}^{\infty}$ for $1 \leq p \leq q<\infty$,

(ii) if $w \in A_{p}^{\infty}$ with $p>1$, then there exists $\epsilon>0$ such that $w \in A_{p-\epsilon}^{\infty}$. Consequently, $A_{p}^{\infty}=\cup_{q<p} A_{q}^{\infty}$.

Similarly, by adapting the ideas to [13], the new BMO space $\mathrm{BMO}_{\theta}$ with $\theta \geq 0$ is defined as a set of all locally integrable functions $b$ satisfying

$$
\frac{1}{|B|} \int_{B}\left|b(y)-b_{B}\right| d y \leq C\left(1+r_{B}\right)^{\theta},
$$

where $B=B\left(x_{B}, r_{B}\right)$ and $b_{B}=(1 /|B|) \int_{B} b$. A norm for $b \in \mathrm{BMO}_{\theta}$, denoted by $\|b\|_{\theta}$, is given by the infimum of the constants satisfying (12). Clearly $\mathrm{BMO}_{\theta_{1}} \subset \mathrm{BMO}_{\theta_{2}}$ for $\theta_{1} \leq \theta_{2}$ and $\mathrm{BMO}_{0}=\mathrm{BMO}$. We define $\mathrm{BMO}_{\infty}=\cup_{\theta>0} \mathrm{BMO}_{\theta}$.

The following result can be considered to be a variant of John-Nirenberg inequality for the spaces $\mathrm{BMO}_{\theta}$.
Proposition 9. Let $\theta>0, s \geq 1$. If $b \in \mathrm{BMO}_{\theta}$, then for all balls $B$

(i)

$$
\left(\frac{1}{|B|} \int_{B}\left|b(y)-b_{B}\right|^{s} d x\right)^{1 / s} \leqslant\|b\|_{\theta}\left(1+r_{B}\right)^{\theta} ;
$$

(ii)

$$
\left(\frac{1}{\left|2^{k} B\right|} \int_{2^{k} B}\left|b(y)-b_{B}\right|^{s} d x\right)^{1 / s} \lesssim\|b\|_{\theta} k\left(1+2^{k} r_{B}\right)^{\theta}
$$

for all $k \in \mathbb{N}$.

The proof is similar (even easier) to [13, Lemma 1 and Proposition 3] and hence we omit details.

2.2. Weighted Estimates for Some Localized Operators. A ball of the form $B\left(x_{B}, r_{B}\right)$ is called a critical ball if $r_{B}=1$. We have the following result.

Proposition 10. There exists a sequence of points $x_{j}, j \geq 1$ in $\mathbb{R}^{n}$ so that the family of critical balls $\left\{Q_{j}\right\}_{j}$ where $Q_{j}:=$ $B\left(x_{j}, 1\right), j \geq 1$ satisfies the following:

(i) $\cup_{j} Q_{j}=\mathbb{R}^{n}$,

(ii) there exists a constant $C$ such that for any $\sigma>1$, $\sum_{j} \chi_{\sigma Q_{j}} \leq C \sigma^{n}$.

Note that the more general version of Proposition 10 is obtained by [14]. However, in our particular situation, for convenience, we would like to give a simple proof of this proposition.

Proof. Let us consider the family of balls $\{B(x, 1 / 5): x \in$ $\left.\mathbb{R}^{n}\right\}$. Using Vitali covering lemma, we can pick the subfamily of balls $\left\{B_{j}:=B\left(x_{j}, 1 / 5\right): j \geq 1\right\}$ so that $\left\{Q_{j}\right\}_{j}$ is pairwise disjoint and $\mathbb{R}^{n} \subset \cup_{j} Q_{j}$ where $Q_{j}=5 B_{j}=B\left(x_{j}, 1\right)$. This gives (i).

To prove (ii), pick any $x \in \mathbb{R}^{n}$. Let $\mathbb{I}$ be the set of all indices $j$ so that $x \in \sigma Q_{j}$. Note that if $x \in \sigma Q_{j}$, then $\sigma Q_{j} \subset B(x, 2 \sigma)$. Therefore, $B\left(x_{j}, 1 / 5\right) \subset B(x, 2 \sigma)$ for all $j \in \mathbb{I}$. Since $\left\{B\left(x_{j}, 1 / 5\right)\right\}_{j \in \mathfrak{J}}$ is pairwise disjoint, $\sum_{j \in \mathfrak{J}}\left|B\left(x_{j}, 1 / 5\right)\right| \leq$ $|B(x, 2 \sigma)|$. This is equivalent to that $|\mathbb{I}| / 5^{n} \leq C \sigma^{n}$. Hence, $|\mathbb{I}| \leq C \sigma^{n}$. This completes our proof.

We consider the following maximal functions for $g \in$ $L_{\mathrm{loc}}^{1}\left(\mathbb{R}^{n}\right)$ and $x \in \mathbb{R}^{n}$ :

$$
\begin{gathered}
M_{\mathrm{loc}, \alpha} g(x)=\sup _{x \in B \in \mathscr{B}_{\alpha}} \frac{1}{|B|} \int_{B}|g|, \\
M_{\mathrm{loc}, \alpha}^{\sharp} g(x)=\sup _{x \in B \in \mathscr{B}_{\alpha}} \frac{1}{|B|} \int_{B}\left|g-g_{B}\right|,
\end{gathered}
$$

where $\mathscr{B}_{\alpha}=\left\{B(y, r): y \in \mathbb{R}^{n}\right.$ and $\left.r \leq \alpha\right\}$. 
Also, given a ball $Q$, we define the following maximal functions for $g \in L_{\mathrm{loc}}^{1}\left(\mathbb{R}^{n}\right)$ and $x \in \mathrm{Q}$ :

$$
\begin{gathered}
M_{\mathrm{Q}} g(x)=\sup _{x \in B \in \mathscr{F}(\mathrm{Q})} \frac{1}{|B \cap Q|} \int_{B \cap Q}|g|, \\
M_{\mathrm{Q}}^{\sharp} g(x)=\sup _{x \in B \in \mathscr{F}(\mathrm{Q})} \frac{1}{|B \cap Q|} \int_{B \cap Q}\left|g-g_{B \cap Q}\right|,
\end{gathered}
$$

where $\mathscr{F}(Q)=\{B(y, r): y \in Q, r>0\}$.

We have the following lemma.

Lemma 11. For $1<p<\infty$, let $\left\{Q_{k}\right\}_{k}$ be a sequence of balls as in Proposition 10. Then

$$
\begin{aligned}
\int_{\mathbb{R}^{n}} \mid & \left.M_{\mathrm{loc}, 1 / 2} g(x)\right|^{p} w(x) d x \\
& \leqslant \int_{\mathbb{R}^{n}}\left|M_{\mathrm{loc}, 4}^{\sharp} g(x)\right|^{p} w(x) d x \\
& \quad+\sum_{k} w\left(Q_{k}\right)\left(\frac{1}{\left|2 Q_{k}\right|} \int_{2 Q_{k}}|g|\right)^{p}
\end{aligned}
$$

for all $g \in L_{\mathrm{loc}}^{1}\left(\mathbb{R}^{n}\right)$ and $w \in A_{\infty}^{\infty}$.

Proof. We adapt the argument in [13, Lemma 2] to our present situation.

By Proposition 10, we have

$$
\begin{aligned}
\int_{\mathbb{R}^{n}}\left|M_{\mathrm{loc}, 1 / 2} g(x)\right|^{p} w(x) d x \\
\quad \leq C \sum_{k} \int_{Q_{k}}\left|M_{\mathrm{loc}, 1 / 2} g(x)\right|^{p} w(x) d x .
\end{aligned}
$$

It can be verified that, for $x \in Q_{k}, M_{\text {loc, } 1 / 2} g(x) \leq$ $M_{2 \mathrm{Q}_{k}}\left(g \chi_{2 \mathrm{Q}_{k}}\right)$. Note that since $g \chi_{2 \mathrm{Q}_{k}}$ is supported in $2 \mathrm{Q}_{k}$, operators $M_{2 Q_{k}}$ and $M_{2 Q_{k}}^{\sharp}$ are Hardy-Littlewood and sharp maximal functions defined in $2 Q_{k}$ viewed as a space of homogeneous type with the Euclidean metric and the Lebesgues measure restricted to $2 Q_{k}$. Moreover, by definition of $A_{\infty}^{\infty}$, if $w \in A_{\infty}^{\infty}$, then $w \in A_{\infty}\left(2 Q_{k}\right)$, where $A_{\infty}\left(2 Q_{k}\right)=$ $\cup_{p \geq 1} A_{p}\left(2 Q_{k}\right)$, and $A_{p}\left(2 Q_{k}\right)$ is the class of Muckenhoupt weights on the spaces of homogeneous type $2 Q_{k}$. Moreover, due to [12, Lemma 5], $[w]_{A_{\infty}\left(2 Q_{k}\right)} \leq C$ for all $k \geq 1$. Therefore, using Proposition 3.4 in [15] gives

$$
\begin{aligned}
\int_{\mathbb{R}^{n}}\left|M_{\mathrm{loc}, 1 / 2} g(x)\right|^{p} w(x) d x \\
\leq C \sum_{k} \int_{\mathrm{Q}_{k}}\left|M_{\mathrm{loc}, 1 / 2} g(x)\right|^{p} w(x) d x \\
\leq C \sum_{k} \int_{\mathrm{Q}_{k}}\left|M_{2 \mathrm{Q}_{k}}\left(g \chi_{2 \mathrm{Q}_{k}}\right)(x)\right|^{p} w(x) d x \\
\leq C \sum_{k} \int_{2 \mathrm{Q}_{k}}\left|M_{2 \mathrm{Q}_{k}}^{\sharp}\left(g \chi_{2 \mathrm{Q}_{k}}\right)(x)\right|^{p} w(x) d x \\
\quad+C \sum_{k} w\left(2 \mathrm{Q}_{k}\right)\left(\frac{1}{\left|2 \mathrm{Q}_{k}\right|} \int_{2 \mathrm{Q}_{k}}|g(x)| d x\right)^{p} .
\end{aligned}
$$

To complete the proof, we need only to check that $M_{2 \mathrm{Q}_{k}}^{\sharp}\left(g \chi_{2 \mathrm{Q}_{k}}\right)(x) \leq C M_{\mathrm{loc}, 4}^{\sharp}(g)(x)$ for $x \in 2 Q_{k}$. We have

$$
M_{\mathrm{loc}, 4}^{\sharp}(g)(x)=\sup _{B \in \mathscr{F}\left(2 Q_{k}\right): B \ni x} \frac{1}{\left|B \cap 2 Q_{k}\right|} \int_{B \cap 2 Q_{k}}\left|f-f_{B \cap 2 Q_{k}}\right| .
$$

If $r_{B} \geq 4$, due to $r_{2 Q_{k}}=2,2 Q_{k} \subset B$. Hence, in this situation, we have

$$
\begin{aligned}
\frac{1}{\left|B \cap 2 Q_{k}\right|} \int_{B \cap 2 Q_{k}}\left|f-f_{B \cap 2 Q_{k}}\right| & =\frac{1}{\left|Q_{k}\right|} \int_{2 Q_{k}}\left|f-f_{2 Q_{k}}\right| \\
& \leq M_{\text {loc }, 4}^{\sharp}(g)(x) .
\end{aligned}
$$

Otherwise, if $r_{B}<4$, it is obvious that $\left|B \cap 2 Q_{k}\right| \approx|B|$. So we have

$$
\begin{aligned}
& \frac{1}{\left|B \cap 2 Q_{k}\right|} \int_{B \cap 2 Q_{k}}\left|f-f_{B \cap 2 Q_{k}}\right| \\
& \quad \leq 2 \frac{1}{\left|B \cap 2 Q_{k}\right|} \int_{B \cap 2 Q_{k}}\left|f-f_{B}\right| \\
& \quad \leq C \frac{1}{|B|} \int_{B}\left|f-f_{B}\right| \leq C M_{\text {loc, } 4}^{\sharp}(g)(x) .
\end{aligned}
$$

This completes our proof.

Let $N>0$. For $\kappa \geq 1$ and $p \geq 1$, we define the following functions for $g \in L_{\mathrm{loc}}^{1}\left(\mathbb{R}^{n}\right)$ and $x \in \mathbb{R}^{n}$ :

$$
\begin{aligned}
& G_{\kappa, p}^{N} f(x) \\
& \quad=\sup _{Q \ni x ; Q} \sum_{\text {is critical }}^{\infty} 2_{k=0}^{-N k}\left(\frac{1}{\left|2^{k} \widehat{Q}\right|} \int_{2^{k} \widehat{Q}}|f(z)|^{p} d z\right)^{1 / p},
\end{aligned}
$$

where $\widehat{Q}=\kappa Q$.

When $\kappa=1$, we write $G_{p}^{N}$ instead of $G_{1, p}^{N}$. The following result gives the weighted estimates for $G_{\kappa, p}$. 
Proposition 12. Let $p>s>1$ and $w \in A_{p / s}^{\theta}, \theta \geq 0$. Then we have

$$
\left\|G_{\kappa, s}^{N} f\right\|_{L^{p}(w)} \lesssim\|f\|_{L^{p}(w)}
$$

provided that $N>\theta / s+n / p$

Without loss of generality, we assume that $\kappa=1$. Assume that $Q=B\left(x_{0}, 1\right)$. For $x \in Q, Q \subset 2 B(x, 1)$. This implies that

$$
\begin{aligned}
& G_{s}^{N} f(x) \\
& \quad \leq C \sum_{k=0}^{\infty} 2^{-N k}\left(\frac{1}{\left|2^{k} B(x, 1)\right|} \int_{B_{k}(x, 1)}|f(z)|^{s} d z\right)^{1 / s},
\end{aligned}
$$

where $B_{k}(x, 1)=B\left(x, 2^{k+1}\right)$.

Let $\left\{Q_{j}\right\}$ be the family of critical balls given by Proposition 10. Note that if $x \in Q_{j}, B_{k}(x, 1) \subset Q_{j}^{k}$ where $Q_{j}^{k}=2^{k+2} Q_{j}$. These estimates and Hölder's inequalities give

$$
\begin{aligned}
& \left\|G_{p}^{N} f\right\|_{L^{p}(w)} \\
& \leq C \sum_{k=0}^{\infty} 2^{-N k}\left(\sum_{j} \int_{Q_{j}}\left(\frac{1}{\left|2^{k} B(x, 1)\right|} \int_{B_{k}(x, 1)}|f(z)|^{s} d z\right)^{p / s}\right. \\
& \times w(x) d x)^{1 / p} \\
& \leq C \sum_{k=0}^{\infty} 2^{-N k} \\
& \times\left(\sum_{j} \int_{Q_{j}}\left(\frac{1}{\left|2^{k} Q_{j}\right|} \int_{Q_{j}^{k}}|f(z)|^{s} d z\right)^{p / s} w(x) d x\right)^{1 / p} \\
& \leq C \sum_{k=0}^{\infty} 2^{-N k}\left(\sum_{j} \frac{w\left(Q_{j}\right)}{\left|2^{k} Q_{j}\right|^{p / s}}\left(\int_{Q_{j}^{k}}|f(z)|^{s} d z\right)^{p / s}\right)^{1 / p} \\
& \leq C \sum_{k=0}^{\infty} 2^{-N k}\left(\sum_{j} \frac{w\left(Q_{j}^{k}\right)}{\left|2^{k} Q_{j}\right|^{p / s}}\left(\int_{Q_{j}^{k}} w^{-(p / s)^{\prime} /(p / s)}\right)^{(p / s) /(p / s)^{\prime}}\right. \\
& \left.\times\left(\int_{Q_{j}^{k}}|f(z)|^{p} w(z) d z\right)\right)^{1 / p} .
\end{aligned}
$$

Since $w \in A_{p / s}^{\theta}$, by definition of the classes $A_{p}^{\theta}$, we have

$$
w\left(Q_{j}^{k}\right)\left(\int_{Q_{j}^{k}} w^{-(p / s)^{\prime} /(p / s)}\right)^{(p / s) /(p / s)^{\prime}} \leq C\left|Q_{j}^{k}\right|^{p / s} 2^{k \theta \times(p / s)}
$$

This together with (26) gives

$$
\begin{aligned}
& \left\|G_{s}^{N} f\right\|_{L^{p}(w)} \\
& \quad \leq C \sum_{k} 2^{-k(N-\theta / s)}\left(\sum_{j} \int_{Q_{j}^{k}}|f(z)|^{p} w(z) d z\right)^{1 / p} \\
& \quad \leq C \sum_{k} 2^{-k(N-\theta / s-n / p)}\|f\|_{L^{p}(w)} \\
& \quad \leq C\|f\|_{L^{p}(w)}
\end{aligned}
$$

This completes our proof.

For a family of balls $\left\{Q_{k}\right\}_{k}$ given by Proposition 10, we define the operator $\widetilde{M}_{s}, s \geq 1$, as

$$
\widetilde{M}_{s} f=\sum_{k} \chi_{\mathrm{Q}_{k}} M_{s}\left(f \chi_{\widetilde{Q}_{k}}\right) \text {, }
$$

where $\widetilde{Q}_{j}=8 Q_{j}$ and $M_{s} f=M\left(|f|^{s}\right)^{1 / s}$ with $M$ being the Hardy-Littlewood maximal function. We have the following result.

Proposition 13. If $p>s>1$ and $w \in A_{p / s}^{\theta}, \theta>0$, then $\widetilde{M}_{s}$ is bounded on $L^{p}(w)$.

Proof. We have

$$
\int_{\mathbb{R}^{n}}\left|\widetilde{M}_{s} f(x)\right|^{p} w(x) d x=\sum_{j} \int_{Q_{j}}\left|M_{s}\left(f \chi_{\widetilde{Q}_{k}}\right)\right|^{p} w(x) d x .
$$

For each $k$, if we consider $\widetilde{Q}_{k}$ as a space of homogeneous type with the Euclidean metric and the Lebesgues measure restricted to $\widetilde{Q}_{k}$, then $w \in A_{p / s}\left(\widetilde{Q}_{k}\right)$. Moreover, it can be verified that

$$
\left\|M_{s}\left(f \chi_{\widetilde{Q}_{k}}\right)\right\|_{L^{p}\left(w, \widetilde{Q}_{k}\right)} \leq C\|f\|_{L^{p}\left(w, \widetilde{Q}_{k}\right)},
$$

and the constant $C$ is independent of $k$.

Therefore, by (ii) of Proposition 10,

$$
\begin{aligned}
\int_{\mathbb{R}^{n}}\left|\widetilde{M}_{s} f(x)\right|^{p} w(x) d x & \leq C \sum_{j} \int_{\widetilde{Q}_{k}}|f(x)|^{p} w(x) d x \\
& \leq C\|f\|_{L^{p}(w)}^{p} .
\end{aligned}
$$

This completes our proof.

\section{Some Kernel Estimates}

Let $\varphi_{0}: \mathbb{R}^{n} \rightarrow \mathbb{R}$ be a smooth radial function which is equal to 1 on the unit ball centered at origin and supported on its concentric double. Set $\varphi(\xi)=\varphi_{0}(\xi)-\varphi_{0}(2 \xi)$ and $\varphi_{k}(\xi)=$ $\varphi\left(2^{-k} \xi\right)$. Then, we have

$$
\sum_{k=0}^{\infty} \varphi_{k}(\xi)=1 \quad \forall \xi \in \mathbb{R}^{n}
$$


and supp $\varphi_{k} \subset\left\{\xi: 2^{k-1} \leq|\xi| \leq 2^{k+1}\right\}$ for all $k \geq 1$. Moreover, for any multi-index $\alpha$ and $N \geq 0$, we have

$$
\left|\partial_{\xi}^{\alpha} \varphi_{k}(\xi)\right| \leq c_{\alpha} 2^{-k|\alpha|} .
$$

Lemma 14. Let $a \in L^{\infty} A_{\rho, \delta}^{m}$ with $m \in \mathbb{R}, \rho \in[0,1]$ and $\delta \in[0,1]$. Let $a_{k}(x, y, \xi)=a(x, y, \xi) \varphi_{k}(\xi)$ for $k \geq 0$.

(a) For each $\ell \geq 0$,

$$
|z|^{\ell}\left|\int a_{k}(x, y, \xi) e^{i\langle z, \xi\rangle} d \xi\right| \leq C 2^{k(n+m-\rho \ell)} .
$$

(b) If $a \in L^{\infty} A_{\rho, \delta}^{m}$ with $m<n(\rho-1)$ and $\rho, \delta \in[0,1]$, then, for each $N>0$, there exist $\epsilon, \epsilon^{\prime}>0$ so that for any ball $B \subset \mathbb{R}^{n}, y, \bar{y} \in B$, and $x \in S_{j}(B), j \geq 2$ so that

$$
\begin{array}{r}
\left|\int a_{k}(x, y, \xi) e^{i\langle x-y, \xi\rangle}-a_{k}(x, \bar{y}, \xi) e^{i\langle x-\bar{y}, \xi\rangle} d \xi\right| \\
\leq C 2^{-j \epsilon}\left(2^{j} r_{B}\right)^{-n} \min \left\{1,\left(2^{j} r_{B}\right)^{-N}\right\} 2^{-k \epsilon^{\prime}} .
\end{array}
$$

(c) If $a \in L^{\infty} A_{1, \delta}^{0}, \delta \in[0,1]$, then there exist $\epsilon, \epsilon^{\prime}>0$ so that for any ball $B \subset \mathbb{R}^{n}, y, \bar{y} \in B$, and $x \in S_{j}(B)$, $j \geq 2$ so that

$$
\begin{aligned}
& \left|\int a_{k}(x, y, \xi) e^{i\langle x-y, \xi\rangle}-a_{k}(x, \bar{y}, \xi) e^{i\langle x-\bar{y}, \xi\rangle} d \xi\right| \\
& \leq C 2^{-j \epsilon}\left(2^{j} r_{B}\right)^{-n} \min \left\{1,\left(2^{j} r_{B}\right)^{-N}\right\}\left(2^{k} r_{B}\right)^{\epsilon^{\prime}}
\end{aligned}
$$

as long as $2^{k} r_{B} \leq 1$; and

$$
\begin{aligned}
& \left|\int a_{k}(x, y, \xi) e^{i\langle x-y, \xi\rangle}-a_{k}(x, \bar{y}, \xi) e^{i\langle x-\bar{y}, \xi\rangle} d \xi\right| \\
& \quad \leq C 2^{-j \epsilon}\left(2^{j} r_{B}\right)^{-n} \min \left\{1,\left(2^{j} r_{B}\right)^{-N}\right\}\left(2^{k} r_{B}\right)^{-\epsilon^{\prime}}
\end{aligned}
$$

as long as $2^{k} r_{B}>1$.

Proof. We refer to Lemma 3.1 in [5] for the proof of (a).

(b) We first note that since $a \in L^{\infty} A_{\rho, \delta}^{m}$, we have

$$
\left|\partial_{\xi}^{\alpha} a_{k}(x, y, \xi)\right| \leq c_{\alpha} 2^{k(m-\rho|\alpha|)} \quad \forall k=1,2, \ldots
$$

Since $x \in S_{j}(B), j \geq 2$ and $y, \bar{y} \in B$, we have $x-y \approx x-\bar{y}$. If $|y-\bar{y}|>2^{-k}$, using (a) with $\ell=n+\epsilon$ so that $m-n(\rho-1)-$ $\rho \epsilon+\epsilon<0$ gives

$$
\begin{aligned}
\text { LHS } & :=\left|\int a_{k}(x, y, \xi) e^{i\langle x-y, \xi\rangle}-a_{k}(x, \bar{y}, \xi) e^{i\langle x-\bar{y}, \xi\rangle} d \xi\right| \\
& \leq\left|\int a_{k}(x, y, \xi) e^{i\langle x-y, \xi\rangle} d \xi\right|+\left|\int a_{k}(x, \bar{y}, \xi) e^{i\langle x-\bar{y}, \xi\rangle} d \xi\right| \\
& \leq C|x-y|^{-n-\epsilon} 2^{k(n+m-\rho n-\rho \epsilon)} \\
& \leq C\left(2^{j} r_{B}\right)^{-n-\epsilon} 2^{k(m-n(\rho-1)-\rho \epsilon)} .
\end{aligned}
$$

This together with the fact that $|y-\bar{y}|>2^{-k}$ gives

$$
\begin{aligned}
\text { LHS } & \leq C\left(2^{j} r_{B}\right)^{-n-\epsilon} 2^{k(m-n(\rho-1)-\rho \epsilon)} \\
& \leq C\left(2^{j} r_{B}\right)^{-n+1} 2^{k((m-n(\rho-1))-\rho \epsilon+\epsilon)}|y-\bar{y}|^{\epsilon} \\
& \leq C 2^{-j \epsilon}\left(2^{j} r_{B}\right)^{-n} 2^{-k \epsilon^{\prime}},
\end{aligned}
$$

where $\epsilon^{\prime}=-[(m-n(\rho-1))-\rho \epsilon+\epsilon]>0$.

If $|y-\bar{y}| \leq 2^{-k}$, we have

$$
\begin{aligned}
\text { LHS } \leq & \left|\int a_{k}(x, y, \xi)\left(1-e^{i\langle y-\bar{y}, \xi\rangle}\right) e^{i\langle x-y, \xi\rangle} d \xi\right| \\
& +\left|\int\left(a_{k}(x, y, \xi)-a_{k}(x, \bar{y}, \xi)\right) e^{i\langle x-\bar{y}, \xi\rangle} d \xi\right| \\
& :=E_{1}+E_{2} .
\end{aligned}
$$

We will claim that, for all $\ell \geq 0$, we have

$$
E_{1} \leq C\left(2^{j} r_{B}\right)^{\ell} 2^{k(m+n-\rho \ell+1)}|y-\bar{y}|
$$

Indeed, we have for all integers $\ell \geq 0$,

$$
\begin{aligned}
E_{1} \leq & |x-y|^{-\ell}|x-y|^{\ell} \\
& \times\left|\int a_{k}(x, y, \xi)\left(1-e^{i\langle y-\bar{y}, \xi\rangle}\right) e^{i\langle x-y, \xi\rangle} d \xi\right| \\
\leq & \left(2^{j} r_{B}\right)^{-\ell} \\
& \times\left|\sum_{|\alpha|=\ell} \int(x-y)^{\alpha} a_{k}(x, y, \xi)\left(1-e^{i\langle y-\bar{y}, \xi\rangle}\right) e^{i\langle x-y, \xi\rangle} d \xi\right| \\
\leq & \left(2^{j} r_{B}\right)^{-\ell} \\
& \times\left|\sum_{|\alpha|=\ell} \int a_{k}(x, y, \xi)\left(1-e^{i\langle y-\bar{y}, \xi\rangle}\right) \partial_{\xi}^{\alpha} e^{i\langle x-y, \xi\rangle} d \xi\right| .
\end{aligned}
$$

Using integration by parts, we get that

$$
\begin{aligned}
E_{1} \leq & \left(2^{j} r_{B}\right)^{-\ell} \\
& \times\left|\sum_{|\alpha|=\ell} \int \partial_{\xi}^{\alpha}\left[a_{k}(x, y, \xi)\left(1-e^{i\langle y-\bar{y}, \xi\rangle}\right)\right] e^{i\langle x-y, \xi\rangle} d \xi\right| .
\end{aligned}
$$

We write

$$
\begin{aligned}
\sum_{|\alpha|=\ell} \partial_{\xi}^{\alpha}\left[a_{k}(x, y, \xi)\left(1-e^{i\langle y-\bar{y}, \xi\rangle}\right)\right] & \\
& =\sum_{|\alpha|+|\beta|=\ell} \partial_{\xi}^{\alpha} a_{k}(x, y, \xi) \partial_{\xi}^{\beta}\left(1-e^{i\langle y-\bar{y}, \xi\rangle}\right) .
\end{aligned}
$$


If $|\beta|=0,\left|1-e^{i\langle y-\bar{y}, \xi\rangle}\right| \leq C|y-\bar{y}||\xi| \leq C 2^{k}|y-\bar{y}|$. Therefore, in this situation,

$$
\begin{aligned}
& \left|\sum_{|\alpha|=\ell} \int \partial_{\xi}^{\alpha}\left[a_{k}(x, y, \xi)\right]\left(1-e^{i\langle y-\bar{y}, \xi\rangle}\right) e^{i\langle x-y, \xi\rangle} d \xi\right| \\
& \leq C 2^{k(n+m+1-\rho|\alpha|)}|y-\bar{y}|=C 2^{k(n+m+1-\rho \ell)}|y-\bar{y}| .
\end{aligned}
$$

Otherwise, $\left|\partial_{\xi}^{\beta}\left(1-e^{i\langle y-\bar{y}, \xi\rangle}\right)\right| \leq C|y-\bar{y}|^{|\beta|}$. This together with (39) gives

$$
\begin{aligned}
& \left|\int \partial_{\xi}^{\alpha} a_{k}(x, y, \xi) \partial_{\xi}^{\beta}\left(1-e^{i\langle y-\bar{y}, \xi\rangle}\right) e^{i\langle x-y, \xi\rangle} d \xi\right| \\
& \quad \leq C 2^{k(n+m-\rho|\alpha|)}|y-\bar{y}|^{|\beta|} \\
& \quad \leq C 2^{k(n+m+1-\rho|\alpha|-|\beta|)}|y-\bar{y}| \\
& \quad \leq C 2^{k(n+m+1-\rho \ell)}|y-\bar{y}| .
\end{aligned}
$$

Therefore,

$$
E_{1} \leq C\left(2^{j} r_{B}\right)^{-\ell} 2^{k(m+n-\rho \ell+1)}|y-\bar{y}| .
$$

The general statement for noninteger values of $\ell$ follows by interpolation of the inequality for $i$ and $i+1$, where $i<\ell<$ $i+1$. Therefore, (43) holds for all $\ell>0$. Now taking $\ell=n+\epsilon$ so that $\epsilon^{\prime}=-(m+n-\rho n-\rho \epsilon+\epsilon)>0$, we have

$$
\begin{aligned}
E_{1} & \leq C\left(2^{j} r_{B}\right)^{-n-\epsilon} 2^{k(m+n-\rho n-\rho \epsilon+\epsilon)}|y-\bar{y}|^{\epsilon}\left(2^{k}|y-\bar{y}|\right)^{1-\epsilon} \\
& \leq C\left(2^{j} r_{B}\right)^{-n-\epsilon} 2^{-k \epsilon^{\prime}}|y-\bar{y}|^{\epsilon} \\
& \leq C 2^{-j \epsilon}\left(2^{j} r_{B}\right)^{-n} 2^{-k \epsilon^{\prime}} .
\end{aligned}
$$

It remains to take care of the term $E_{2}$. Repeating the previous arguments we also obtain

$$
\begin{aligned}
E_{2} \leq & \left(2^{j} r_{B}\right)^{-\ell} \\
& \times\left|\sum_{|\alpha|=\ell} \int \partial_{\xi}^{\alpha}\left[a_{k}(x, y, \xi)-a_{k}(x, \bar{y}, \xi)\right] e^{i\langle y-\bar{y}, \xi\rangle} d \xi\right| .
\end{aligned}
$$

At this stage, using the mean value theorem (applied for each component of $a_{k}$ ) and then using the definition of the class $L^{\infty} A_{\rho, \delta}^{m}$ give

$$
\begin{aligned}
E_{2} & \leq C\left(2^{j} r_{B}\right)^{-\ell}|y-\bar{y}| 2^{k(n+m-\rho \ell+\delta)} \\
& \leq C\left(2^{j} r_{B}\right)^{-\ell}|y-\bar{y}| 2^{k(n+m-\rho \ell+1)}
\end{aligned}
$$

for all integer $\ell \geq 0$. Hence, by interpolation again,

$$
E_{2} \leq C\left(2^{j} r_{B}\right)^{-\ell}|y-\bar{y}| 2^{k(n+m-\rho \ell+1)}
$$

for all $\ell \geq 0$. Repeating the arguments used to estimate $E_{1}$, we conclude that

$$
E_{2} \leq C 2^{-j \epsilon}\left(2^{j} r_{B}\right)^{-n} 2^{-k \epsilon^{\prime}}
$$

Therefore, LHS $\leq C 2^{-j \epsilon}\left(2^{j} r_{B}\right)^{-n} 2^{-k \epsilon^{\prime}}$. It remains to show that

$$
\text { LHS } \leq C 2^{-j \epsilon}\left(2^{j} r_{B}\right)^{-n-N} 2^{-k \epsilon^{\prime}} .
$$

To do this, we repeat the arguments above with $\ell=N+n+\epsilon$. Since the proof of this part is analogous to (55), and hence we omit details here. This completes our proof.

(c) If $2^{-k} \leq r_{B}$, using the argument as in (b), we have

$$
\begin{aligned}
\text { LHS } & :=\left|\int a_{k}(x, y, \xi) e^{i\langle x-y, \xi\rangle}-a_{k}(x, \bar{y}, \xi) e^{i\langle x-\bar{y}, \xi\rangle} d \xi\right| \\
& \leq\left|\int a_{k}(x, y, \xi) e^{i\langle x-y, \xi\rangle} d \xi\right|+\left|\int a_{k}(x, \bar{y}, \xi) e^{i\langle x-\bar{y}, \xi\rangle} d \xi\right| \\
& \leq C|x-y|^{-n-\epsilon} 2^{-k \epsilon} \\
& \leq C \frac{\left(2^{j} r_{B}\right)^{-n-\epsilon}}{r_{B}^{\epsilon}} \frac{2^{-k \epsilon}}{r_{B}^{\epsilon}}=C 2^{-j \epsilon}\left(2^{j} r_{B}\right)^{n}\left(\frac{1}{r_{B} 2^{k}}\right)^{\epsilon} .
\end{aligned}
$$

If $r_{B}<2^{-k}$, we have

$$
\begin{aligned}
\text { LHS } \leq & \left|\int a_{k}(x, y, \xi)\left(1-e^{i\langle y-\bar{y}, \xi\rangle}\right) e^{i\langle x-y, \xi\rangle} d \xi\right| \\
& +\left|\int\left(a_{k}(x, y, \xi)-a_{k}(x, \bar{y}, \xi)\right) e^{i\langle x-\bar{y}, \xi\rangle} d \xi\right| \\
& :=E_{1}+E_{2} .
\end{aligned}
$$

The previous arguments in (b) show that

$$
\begin{aligned}
E_{1}+E_{2} & \leq C\left(2^{j} r_{B}\right)^{-n-\epsilon} 2^{k(-\epsilon+1)}|y-\bar{y}| \\
& \leq C\left(2^{j} r_{B}\right)^{-n-\epsilon} 2^{k(-\epsilon+1)} r_{B} \\
& =C\left(2^{j} r_{B}\right)^{-n-\epsilon} r_{B}^{\epsilon}\left(r_{B} 2^{k}\right)^{(-\epsilon+1)} \\
& \leq C 2^{-j \epsilon}\left(2^{j} r_{B}\right)^{-n}\left(r_{B} 2^{k}\right)^{(1-\epsilon)}
\end{aligned}
$$

Hence,

$$
\begin{gathered}
\left|\int a_{k}(x, y, \xi) e^{i\langle x-y, \xi\rangle}-a_{k}(x, \bar{y}, \xi) e^{i\langle x-\bar{y}, \xi\rangle} d \xi\right| \\
\leq C 2^{-j \epsilon}\left(2^{j} r_{B}\right)^{-n}\left(2^{k} r_{B}\right)^{\epsilon^{\prime}} \text { if } 2^{k} r_{B} \leq 1, \\
\left|\int a_{k}(x, y, \xi) e^{i\langle x-y, \xi\rangle}-a_{k}(x, \bar{y}, \xi) e^{i\langle x-\bar{y}, \xi\rangle} d \xi\right| \\
\leq C 2^{-j \epsilon}\left(2^{j} r_{B}\right)^{-n}\left(2^{k} r_{B}\right)^{-\epsilon} \text { if } 2^{k} r_{B}>1 .
\end{gathered}
$$


By taking $\ell=n+N+\epsilon$ and repeating the previous arguments, we obtain that

$$
\begin{gathered}
\left|\int a_{k}(x, y, \xi) e^{i\langle x-y, \xi\rangle}-a_{k}(x, \bar{y}, \xi) e^{i\langle x-\bar{y}, \xi\rangle} d \xi\right| \\
\leq C 2^{-j \epsilon}\left(2^{j} r_{B}\right)^{-n-N}\left(2^{k} r_{B}\right)^{\epsilon^{\prime}} \text { if } 2^{k} r_{B} \leq 1, \\
\left|\int a_{k}(x, y, \xi) e^{i\langle x-y, \xi\rangle}-a_{k}(x, \bar{y}, \xi) e^{i\langle x-\bar{y}, \xi\rangle} d \xi\right| \\
\leq C 2^{-j \epsilon}\left(2^{j} r_{B}\right)^{-n-N}\left(2^{k} r_{B}\right)^{-\epsilon^{\prime}} \text { if } 2^{k} r_{B}>1 .
\end{gathered}
$$

This completes the proof of (c).

Since the associated kernel $K(x, y)$ of the operator $T_{a}$ is given by

$$
\begin{aligned}
K(x, y) & =\frac{1}{(2 \pi)^{n}} \int a(x, y, \xi) e^{i\langle x-y, \xi\rangle} d \xi \\
& =\sum_{k \geq 0} \frac{1}{(2 \pi)^{n}} \int a_{k}(x, y, \xi) e^{i\langle x-y, \xi\rangle} d \xi
\end{aligned}
$$

with $a_{k}(x, \xi)$ as in Lemma 14 , from Lemma 14 we deduce directly the following result.

Lemma 15. Let $a \in L^{\infty} A_{\rho, \delta}^{m}$ with $m<n(\rho-1)$ or $a \in L^{\infty} A_{1, \delta}^{0}$, $\delta \in[0,1]$, and let $K^{*}(x, y)$ be the associated kernel of the operator $T_{a}^{*}$, the conjugate of $T_{a}$.

(a) For any $N>0$, we have

$$
\left|K^{*}(x, y)\right| \leq \frac{C}{|x-y|^{-N}}, \quad x \neq y .
$$

(b) For any $N>0$, there exists $\epsilon>0$ so that any ball $B C$ $\mathbb{R}^{n}, y, \bar{y} \in B, x \in S_{j}(B), j \geq 2$, we have

$$
\begin{aligned}
& \left|K^{*}(y, x)-K^{*}(\bar{y}, x)\right| \\
& \quad \leq C 2^{-j \epsilon}\left(2^{j} r_{B}\right)^{-n} \min \left\{1,\left(2^{j} r_{B}\right)^{-N}\right\} .
\end{aligned}
$$

\section{Proof of Theorem 3}

Note that, by duality argument, the linear operator $T$ is bounded on $L^{p}(w), 1<p<\infty$ if and only if its conjugate $T^{*}$ is bounded on $L^{p^{\prime}}\left(w^{1-p^{\prime}}\right)$. Moreover, by Hölder's inequality, it can be verified that $w \in A_{p}^{\infty}$ if and only if $w^{1-p^{\prime}} \in A_{p^{\prime}}^{\infty}$. Therefore, it suffices to prove (a) and (b) for $T_{a}^{*}$ and $T_{a}^{*, b}=$ $\left[b, T_{a}^{*}\right]$ with $b \in \mathrm{BMO}_{\infty}$. Before coming to the proof of Theorem 3, we need the following results.

Lemma 16. Let $a \in L^{\infty} A_{\rho, \delta}^{m}$ with $m<n(\rho-1)$ or $a \in L^{\infty} A_{1, \delta}^{0}$, $\delta \in[0,1]$, and $b \in B M O_{\theta}, \theta \geq 0$. If $T_{a}$ is bounded on $L^{p}$ for all $1<p<\infty$, then for any $p>1$ and $N>0$ there exists $C>0$ such that for all balls $Q=Q\left(x_{0}, 1\right)$, (a)

$$
\frac{1}{|Q|} \int_{Q}\left|T_{a}^{*} f(x)\right| d x \leq \operatorname{Cinf}_{y \in Q} G_{p}^{N}(y)
$$

(b)

$$
\frac{1}{|Q|} \int_{Q}\left|T_{a}^{*, b} f(x) d x\right| \leq C \inf _{y \in Q} G_{p}^{N-n-\theta} f(y)\|b\|_{\theta}
$$

Proof. (a) We split $f=f_{1}+f_{2}$ where $f_{1}=f \chi_{4 Q}$. For each $j \geq 0$, we have

$$
\begin{aligned}
\frac{1}{|Q|} \int_{Q}\left|T_{a}^{*} f(x)\right| d x & \leq \frac{1}{|Q|} \int_{Q}\left|T_{a}^{*} f_{1}\right|+\frac{1}{|Q|} \int_{Q}\left|T_{a}^{*} f_{2}\right| \\
& :=I_{1}+I_{2} .
\end{aligned}
$$

Using Hölder's inequality and the fact that $T_{a}^{*}$ is bounded on $L^{p}, 1<p<\infty$, we write

$$
\begin{aligned}
I_{1} & \leq C\left(\frac{1}{|Q|} \int_{Q}\left|T_{a}^{*} f_{1}\right|^{p}\right)^{1 / p} \leq\left(\frac{1}{|4 Q|} \int_{4 Q}|f|^{p}\right)^{1 / p} \\
& \leq C \inf _{y \in Q} G_{p}^{N} f(y) .
\end{aligned}
$$

For the term $I_{2}$ we have, for $x \in Q$,

$$
\begin{aligned}
T_{a}^{*} f_{2}(x) & =\int_{R^{n} \backslash 4 Q} K^{*}(x, y) f(y) d y \\
& =\int_{R^{n} \backslash 4 Q} K^{*}(y, x) f(y) d y \\
& =\sum_{k \geq 3} \int_{S_{k}(Q)} K^{*}(y, x) f(y) d y .
\end{aligned}
$$

Applying (a) of Lemma 15, we have

$$
\begin{aligned}
T_{a}^{*} f_{2}(x) & =\sum_{k \geq 3} \int_{S_{k}(Q)} K^{*}(x, y) f(y) d y \\
& \leq \sum_{k \geq 3} \int_{S_{k}(Q)} \frac{f(y)}{|x-y|^{n+N}} d y \\
& \leq \operatorname{Cinf}_{y \in Q} G^{N} f(y) \leq C \inf _{y \in Q} G_{p}^{N} f(y) .
\end{aligned}
$$

This completes the proof of (a).

(b) Taking $1<r<p$, we write

$$
T_{a}^{*, b} f=\left(b-b_{Q}\right) T_{a}^{*} f-T_{a}^{*}\left(\left(b-b_{Q}\right) f\right) .
$$

So, we have

$$
\begin{aligned}
\frac{1}{|Q|} \int_{Q}\left|T_{a}^{*, b} f(x)\right| d x \leq & \frac{1}{|Q|} \int_{Q}\left|\left(b-b_{Q}\right) T_{a}^{*} f\right| d x \\
& +\frac{1}{|Q|} \int_{Q}\left|T_{a}^{*}\left(\left(b-b_{Q}\right) f\right)(x)\right| d x \\
:= & I_{1}+I_{2} .
\end{aligned}
$$


We now take care of $I_{1}$. By Hölder's inequality, we can write

$$
\begin{aligned}
I_{1} & \leq C\|b\|_{\theta}\left(\frac{1}{|Q|} \int_{Q}\left|T_{a}^{*} f\right|^{p}\right)^{1 / p} \\
& \leq C\|b\|_{\theta}\left(\left(\frac{1}{|Q|} \int_{Q}\left|T_{a}^{*} f_{1}\right|^{p}\right)^{1 / p}+\left(\frac{1}{|Q|} \int_{Q}\left|T_{a}^{*} f_{2}\right|^{p}\right)^{1 / p}\right) \\
& :=I_{11}+I_{12},
\end{aligned}
$$

where $f=f_{1}+f_{2}$ with $f_{1}=f \chi_{4 Q}$.

Due to $L^{p}$ boundedness of $T_{a}^{*}$, one has

$$
I_{11} \leq C\left(\frac{1}{|4 Q|} \int_{4 Q}|f|^{p}\right)^{1 / p} \leq \operatorname{Cinf}_{y \in Q} G_{p}^{N} f(y) .
$$

To estimate $I_{12}$, using (69) gives $I_{12} \leq C \inf _{y \in Q} G_{p}^{N} f(y)$.

The estimate for $I_{2}$ can be proceeded in the same method. Indeed, we write

$$
\begin{aligned}
\frac{1}{|Q|} \int_{Q}\left|T_{a}^{*}\left(\left(b-b_{Q}\right) f\right)(x)\right| d x \\
\leq \frac{1}{|Q|} \int_{Q}\left|T_{a}^{*}\left(\left(b-b_{Q}\right) f_{1}\right)(x)\right| d x \\
\quad+\frac{1}{|Q|} \int_{Q}\left|T_{a}^{*}\left(\left(b-b_{Q}\right) f_{2}\right)(x)\right| d x \\
:=I_{21}+I_{22},
\end{aligned}
$$

where $f=f_{1}+f_{2}$ and $f_{1}=f \chi_{4 Q}$.

To estimate $I_{21}$, using Hölder's inequality, we have

$$
\begin{aligned}
\frac{1}{|Q|} \int_{Q}\left|T_{a}^{*}\left(\left(b-b_{Q}\right) f_{1}\right)(x)\right| d x \\
\leq\left(\frac{1}{|Q|} \int_{Q}\left|T_{a}^{*}\left(\left(b-b_{Q}\right) f_{1}\right)(x)\right|^{r} d x\right)^{1 / r} \\
\leq\left(\frac{1}{|Q|} \int_{Q}\left|\left(\left(b-b_{Q}\right) f_{1}\right)(x)\right|^{r} d x\right)^{1 / r} \\
\leq\left(\frac{1}{|4 Q|} \int_{4 Q}|f(x)|^{p} d x\right)^{1 / p} \\
\quad \times\left(\frac{1}{|4 Q|} \int_{4 Q}\left|b(x)-b_{Q}\right|^{v} d x\right)^{1 / v} \quad\left(v=\frac{p r}{p-r}\right) \\
\leq C\|b\|_{\theta} \inf _{y \in Q} G_{p}^{N} f(y) .
\end{aligned}
$$

For the term $I_{22}$, due to (a) of Lemma 15, we can write

$$
\begin{aligned}
& T_{a}^{*}\left(\left(b-b_{Q}\right) f_{2}\right)(x) \\
& \quad=\sum_{k \geq 3} \int_{S_{k}(Q)} K^{*}(x, y)\left(\left(b-b_{Q}\right) f\right)(y) d y \\
& \quad \leq C \sum_{k \geq 3} 2^{-k N} \int_{S_{k}(Q)}\left|\left(b(y)-b_{Q}\right) f(y)\right| d y .
\end{aligned}
$$

By Hölder's inequality and Proposition 9, we give

$$
\begin{aligned}
\int_{S_{k}(\mathrm{Q})} & \left|\left(b(y)-b_{\mathrm{Q}}\right) f(y)\right| d y \\
\leq & \left|2^{k} \mathrm{Q}\right|\left(\frac{1}{\left|2^{k} \mathrm{Q}\right|} \int_{2^{k} \mathrm{Q}}|f|^{p}\right)^{1 / p} \\
& \times\left(\frac{1}{\left|2^{k} \mathrm{Q}\right|} \int_{2^{k} \mathrm{Q}}\left|b-b_{\mathrm{Q}}\right|^{p^{\prime}}\right)^{1 / p^{\prime}} \\
\leq & k 2^{k \theta}\left|2^{k} \mathrm{Q}\right|\|b\|_{\theta}\left(\frac{1}{\left|2^{k} \mathrm{Q}\right|} \int_{2^{k} \mathrm{Q}}^{|f|^{p}}\right)^{1 / p} \\
\leq & k 2^{k(\theta+n)}\|b\|_{\theta}\left(\frac{1}{\left|2^{k} \mathrm{Q}\right|} \int_{2^{k} \mathrm{Q}}|f|^{p}\right)^{1 / p} .
\end{aligned}
$$

From (77) and (76) we obtain that

$$
T_{a}^{*}\left(\left(b-b_{\mathrm{Q}}\right) f_{2}\right)(x) \leq C\|b\|_{\theta} \inf _{y \in Q} G_{p}^{N-n-\theta} f(y) .
$$

This completes our proof.

Remark 17. The result in Lemma 16 still holds if we replace the critical ball $Q$ by $2 Q$.

Lemma 18. Let $a \in L^{\infty} A_{\rho, \delta}^{m}$ with $m<n(\rho-1)$ or $a \in L^{\infty} A_{1, \delta}^{0}$, $\delta \in[0,1]$ and $b \in \mathrm{BMO}_{\theta}, \theta \geq 0$. If $T_{a}$ is bounded on $L^{p}$ for all $1<p<\infty$, then for any $p>1$ and $N>0$ there exists $C>0$ so that, for all $f$ and $x, y \in B=B\left(x_{B}, r_{B}\right)$ with $r_{B}<4$, we have

(a)

$$
\begin{array}{r}
\int_{\mathbb{R}^{n} \backslash 2 B}\left|\left(K^{*}(x, z)-K^{*}(y, z)\right) f(z)\right| d z \\
\leq C\left(\inf _{u \in B} G_{4, p}^{N} f(u)+\inf _{u \in B} \widetilde{M}_{p} f(u)\right) ;
\end{array}
$$

(b)

$$
\begin{array}{r}
\int_{\mathbb{R}^{n} \backslash 2 B}\left|\left(K^{*}(x, z)-K^{*}(y, z)\right)\left(\left(b-b_{B}\right) f\right)(z)\right| d z \\
\leq C\|b\|_{\theta} \inf _{u \in B}\left(\inf _{u \in B} G_{4, p}^{N-n-\theta} f(u)+\inf _{u \in B} \widetilde{M}_{p} f(u)\right) .
\end{array}
$$


Proof. (a) Using (b) of Lemma 15, we write

$$
\begin{aligned}
\int_{\mathbb{R}^{n} \backslash 2 B}\left|\left(K^{*}(x, z)-K^{*}(y, z)\right) f(z)\right| d z \\
\leq C \sum_{k \geq 2} \int_{S_{k}(B)}\left|\left(K^{*}(x, z)-K^{*}(y, z)\right) f(z)\right| d z \\
\leq C \sum_{k \geq 2} 2^{-k \epsilon}\left(2^{k} r_{B}\right)^{-n} \min \left\{1,\left(2^{j} r_{B}\right)^{-N}\right\} \int_{S_{k}(B)}|f(z)| d z \\
\leq C \sum_{k \geq 2} 2^{-k \epsilon} \min \left\{1,\left(2^{j} r_{B}\right)^{-N}\right\} \frac{1}{\left|2^{k} B\right|} \int_{S_{k}(B)}|f(z)| d z \\
=\sum_{k=2}^{k_{0}} \cdots+\sum_{k>k_{0}} \ldots:=I_{1}+I_{2},
\end{aligned}
$$

where $k_{0}$ is the smallest integer so that $2^{k_{0}+1} r_{B}>4$.

To estimate $I_{1}$, let $\left\{Q_{l}\right\}$ and $\left\{\widetilde{Q}_{l}\right\}$ be families of balls as in (29). If $x \in Q_{l} \cap B$, then $2^{k} B \subset \widetilde{Q}_{l}$ for all $k=1,2, \ldots, k_{0}$. This implies that

$$
\frac{1}{\left|2^{k} B\right|} \int_{2^{k} B}|f(z)| d z \leq \inf _{u \in B} \widetilde{M}_{p} f(u)
$$

for all $k=1,2, \ldots, k_{0}$.

Hence

$$
I_{1} \leq \sum_{k=2}^{k_{0}} 2^{-k \epsilon} \inf _{u \in B} \widetilde{M}_{p} f(u) \leq C \inf _{u \in B} \widetilde{M}_{p} f(u) .
$$

For the term $I_{2}$, since $2^{k_{0}} r_{B} \geq 4$ we have

$$
\begin{aligned}
I_{2} & \leq \sum_{k \geq k_{0}} 2^{-k \epsilon}\left(2^{k} r_{B}\right)^{-N} \frac{1}{\left|2^{k} B\right|} \int_{S_{k}(B)}|f(z)| d z \\
& \leq \sum_{k \geq k_{0}} 2^{-k \epsilon}\left(2^{k-k_{0}} 2^{k_{0}} r_{B}\right)^{-N} \frac{1}{\left|2^{k-k_{0}} 2^{k_{0}} B\right|} \int_{2^{k-k_{0} 2^{k_{0} B}}}|f(z)| d z \\
& \leq \sum_{k \geq k_{0}} 2^{-k \epsilon}\left(2^{k-k_{0}}\right)^{-N} \frac{1}{\left|2^{k-k_{0}} 2^{k_{0}} B\right|} \int_{2^{k-k_{0}} 2^{k_{0} B}}|f(z)| d z \\
& \leq \sum_{k \geq 0} 2^{-k \epsilon} 2^{-k N} \frac{1}{\left|2^{k} 2^{k_{0}} B\right|} \int_{2^{k} 2^{k_{0} B}}|f(z)| d z .
\end{aligned}
$$

Note that $2^{k_{0}} B \subset \widehat{Q}=4 Q$ here $Q=B\left(x_{0}, 1\right)$ and $|Q| \approx\left|2^{k_{0}} B\right|$. So, we have

$$
\begin{aligned}
I_{2} & \leq \sum_{k \geq 0} 2^{-k \epsilon} 2^{-k N}\left(\frac{1}{\left|2^{k} \widehat{Q}\right|} \int_{2^{k} \widehat{Q}}|f(z)| d z\right) \\
& \leq C \inf _{u \in B} G_{4, p}^{N} f(u) .
\end{aligned}
$$

Hence, we get (a). (b) Using Hölder's inequality and (b) of Lemma 15, we obtain that

$$
\begin{aligned}
\int_{\mathbb{R}^{n} \backslash 2 B}\left|\left(K^{*}(x, z)-K^{*}(y, z)\right)\left(\left(b-b_{B}\right) f\right)(z)\right| d z \\
=\sum_{k \geq 2} \int_{S_{k}(B)}\left|\left(K^{*}(x, z)-K^{*}(y, z)\right)\left(\left(b-b_{B}\right) f\right)(z)\right| d z \\
\leq C \sum_{k \geq 2} 2^{-k \epsilon} \min \left\{1,\left(2^{j} r_{B}\right)^{-N}\right\} \frac{1}{\left|2^{k} B\right|} \\
\quad \times \int_{S_{k}(B)}\left|\left(\left(b-b_{B}\right) f\right)(z)\right| d z \\
\leq C \sum_{k \geq 2} 2^{-k \epsilon} \min \left\{1,\left(2^{j} r_{B}\right)^{-N}\right\}\left(\frac{1}{\left|2^{k} B\right|} \int_{2^{k} B}|f(z)|^{p} d z\right)^{1 / p} \\
\quad \times\left(\frac{1}{\left|2^{k} B\right|} \int_{2^{k} B}\left|b(z)-b_{B}\right|^{p^{\prime}} d z\right)^{1 / p^{\prime}} \cdot
\end{aligned}
$$

Now using Proposition 9, we get that

$$
\begin{aligned}
\int_{\mathbb{R}^{n} \backslash 2 B}\left|\left(K^{*}(x, z)-K^{*}(y, z)\right)\left(\left(b-b_{B}\right) f\right)(z)\right| d z \\
\leq C \sum_{k \geq 2} k 2^{-k \epsilon}\left(2^{j} r_{B}\right)^{\theta} \min \left\{1,\left(2^{j} r_{B}\right)^{-N}\right\}\|b\|_{\theta} \\
\times\left(\frac{1}{\left|2^{k} B\right|} \int_{2^{k} B}|f(z)|^{p} d z\right)^{1 / p} .
\end{aligned}
$$

At this stage, repeating the same argument as in (a), we complete the proof of (b).

We are now in position to prove Theorem 3.

Proof of Theorem 3. (a) Using the standard argument, see, for example, [13], fix $1<p<\infty$ and $w \in A_{p}^{\infty}$. Let $N>0$ which will be fixed later. So, by Proposition 8 , we can pick $r>1$ and $v \geq 0$ so that $w \in A_{p / r}^{v}$. By Lemma 11 we have

$$
\begin{aligned}
\left\|T_{a}^{*} f\right\|_{L^{p}(w)}^{p} \leq & \left\|M_{\mathrm{loc}, \beta} T_{a}^{*} f\right\|_{L^{p}(w)}^{p} \\
\leq & C\left\|M_{\mathrm{loc}, 4}^{\sharp} T_{a}^{*} f\right\|_{L^{p}(w)}^{p} \\
& +C \sum_{k} w\left(Q_{k}\right)\left(\frac{1}{2 Q_{k}} \int_{2 Q_{k}}\left|T_{a}^{*} f\right|\right)^{p} \\
:= & I_{1}+I_{2} .
\end{aligned}
$$

Let us estimate $I_{1}$ first. By Lemma 16 and Remark 17, we have

$$
\frac{1}{2 Q_{k}} \int_{2 Q_{k}}\left|T_{a}^{*} f\right| \leq C \inf _{y \in Q_{k}} G_{r}^{N} f(y) .
$$


Invoking Proposition 12, we conclude that

$$
\begin{aligned}
& \sum_{k} w\left(Q_{k}\right)\left(\frac{1}{2 Q_{k}} \int_{2 Q_{k}}\left|T_{a}^{*} f\right|\right)^{p} \\
& \leq \sum_{k} \int_{Q_{k}}\left|G_{r}^{N} f(x)\right|^{p} w(x) d x \\
& \leq C \int_{\mathbb{R}^{n}}\left|G_{r}^{N} f(x)\right|^{p} w(x) d x \\
& \leq C\|f\|_{L^{p}(w)}^{p}
\end{aligned}
$$

as long as $N>n / p+v / r$. We now take care of $I_{2}$. For any ball $B\left(x_{0}, r_{B}\right)$ with $r_{B} \leq 4$ and $x \in B$, we write

$$
\begin{aligned}
\frac{1}{|B|} \int_{B}\left|T_{a}^{*} f(x)-\left(T_{a}^{*} f\right)_{B}\right| d x \\
\leq \frac{2}{|B|} \int_{B}\left|T_{a}^{*} f_{1}(x)\right| d x \\
\quad+\frac{1}{|B|} \int_{B}\left|T_{a}^{*} f_{2}(x)-\left(T_{a}^{*} f_{2}\right)_{B}\right| d x \\
:=E_{1}+E_{2},
\end{aligned}
$$

Due to Lemma 18, we can write

$$
\begin{aligned}
E_{2} \leq & \frac{1}{|B|^{2}} \\
& \times \iint_{B}\left(\int_{\mathbb{R}^{n} \backslash 2 B}\left|\left(K^{*}(u, z)-K^{*}(y, z)\right) f(z)\right| d z\right) d y d u \\
\leq & C\left(\inf _{u \in B} G_{4, r}^{N} f(u)+\inf _{u \in B} \widetilde{M}_{r} f(u)\right) .
\end{aligned}
$$

These two estimates of $E_{1}$ and $E_{2}$ tell us that

$$
M_{\mathrm{loc}, 4}^{\sharp} T_{a}^{*} f(x) \leq C\left(G_{4, r}^{N} f(x)+\widetilde{M}_{r} f(x)\right) .
$$

Applying Proposition 12 and the weighted estimates of $\widetilde{M}_{r}$, we get that

$$
\left\|M_{\mathrm{loc}, 4}^{\sharp} T_{a}^{*} f\right\|_{L^{p}(w)} \leq C\|f\|_{L^{p}(w)}
$$

provided that $M>n / p+v / r$.
From (90) and (95), we obtain that

$$
\left\|T_{a}^{*} f\right\|_{L^{p}(w)} \leq C\|f\|_{L^{p}(w)}
$$

This completes our proof.

(b) Fixed $1<p<\infty, b \in \mathrm{BMO}_{\theta}, \theta \geq 0$ and $w \in A_{p}^{\infty}$. So, we can pick $r>1$ and $v \geq 0$ so that $w \in A_{p / r}^{v}$. Then we have by Lemma 11

$$
\begin{aligned}
\left\|T_{a}^{*, b} f\right\|_{L^{p}(w)}^{p} \leq & \int_{\mathbb{R}^{n}}\left|M_{\mathrm{loc}, \beta}\left(T_{a}^{*, b} f\right)(x)\right|^{p} w(x) d x \\
\leq & C \int_{\mathbb{R}^{n}}\left|M_{\mathrm{loc}, 4}^{\sharp}\left(T_{a}^{*, b} f\right)(x)\right|^{p} w(x) d x \\
& +\sum_{k} w\left(Q_{k}\right)\left(\frac{1}{\left|2 Q_{k}\right|} \int_{2 Q_{k}}\left|T_{a}^{*, b} f\right|\right)^{p},
\end{aligned}
$$

where $\left\{Q_{k}\right\}$ is a family of critical balls given in Lemma 11 .

The analogous argument to that in (a) gives

$$
\begin{gathered}
\sum_{k} w\left(Q_{k}\right)\left(\frac{1}{\left|2 Q_{k}\right|} \int_{2 Q_{k}}\left|T_{a}^{*, b} f\right|\right)^{p} \\
\leq C\|b\|_{\theta}^{p}\|f\|_{L^{p}(w)}^{p} .
\end{gathered}
$$

It remains to estimate $\int_{\mathbb{R}^{n}}\left|M_{\mathrm{loc}, 4}^{\sharp}\left(T_{a}^{*, b} f\right)(x)\right|^{p} w(x) d x$. For any ball $B\left(x_{0}, r_{B}\right)$ with $r_{B} \leq 4$ and $x \in B$, we write

$$
\begin{aligned}
\frac{1}{|B|} \int_{B}\left|T_{a}^{*, b} f(x)-\left(T_{a}^{*, b} f\right)_{B}\right| d x \\
\leq \frac{2}{|B|} \int_{B}\left|\left(b-b_{B}\right) T_{a}^{*} f(x)\right| d x \\
\quad+\frac{2}{|B|} \int_{B}\left|T_{a}^{*}\left(\left(b-b_{B}\right) f_{1}\right)(x)\right| d x \\
\quad+\frac{1}{|B|} \int_{B} \mid T_{a}^{*}\left(\left(b-b_{B}\right) f_{2}\right)(x) \\
\quad-\left(T_{a}^{*}\left(\left(b-b_{B}\right) f_{2}\right)\right)_{B} \mid d x \\
=E_{1}+E_{2}+E_{3},
\end{aligned}
$$

where $f=f_{1}+f_{2}$ with $f_{1}=f \chi_{2 B}$.

Hölder's inequality and Proposition 9 show that

$$
\begin{aligned}
E_{1} & \leq C\left(\frac{1}{|B|} \int_{B}\left|b-b_{B}\right|^{r^{\prime}}\right)^{1 / r^{\prime}}\left(\frac{1}{|B|} \int_{B}\left|T_{a}^{*}\right|^{r}\right)^{1 / r} \\
& \leq C\|b\|_{\theta}\left(\frac{1}{|B|} \int_{B}\left|T_{a}^{*} f\right|^{r}\right)^{1 / r} \cdot
\end{aligned}
$$

For any critical ball $Q_{j}$ such that $x \in Q_{j} \cap B$. It can be verified that $B \subset \widetilde{Q}_{j}:=8 Q_{j}$. This yields that

$$
E_{1} \leq C\|b\|_{\theta} \times \inf _{y \in B} \widetilde{M}_{r}\left(T_{a}^{*} f\right)(y) .
$$


Using Hölder's inequality and Proposition 9 again, we have, for $1<s<r$,

$$
\begin{aligned}
E_{2} & \leq C\left(\frac{1}{|B|} \int_{B}\left|T_{a}^{*}\left(\left(b-b_{B}\right) f_{1}\right)\right|^{s}\right)^{1 / s} \\
& \leq C\left(\frac{1}{|B|} \int_{2 B}\left|\left(b-b_{B}\right) f_{1}\right|^{s}\right)^{1 / s} \\
& \leq\left(\frac{1}{|B|} \int_{2 B}\left|\left(b-b_{B}\right)\right|^{\gamma}\right)^{1 / \gamma}\left(\frac{1}{|B|} \int_{2 B}|f|^{r}\right)^{1 / r} \\
& \leq\|b\|_{\theta} \times \inf _{y \in B} \widetilde{M}_{r}(f)(y) .
\end{aligned}
$$

To estimate $E_{3}$, using Lemma 18 , we conclude that

$$
\begin{gathered}
E_{3} \leq C \frac{1}{|B|^{2}} \iint_{B}\left(\int_{R^{n} \backslash 2 B}\left|K^{*}(u, z)-K^{*}(y, z)\right|\right. \\
\left.\quad \times\left|b(z)-b_{B}\right||f(z)| d z\right) d y d u \\
\leq C\|b\|_{\theta}\left(G_{4, r}^{N-\theta-n} f(x)+\widetilde{M}_{r}(f)(x)\right) .
\end{gathered}
$$

These three estimates of $E_{1}, E_{2}$, and $E_{3}$ give

$$
\begin{aligned}
M_{\mathrm{loc}, 4}^{\sharp}\left(T_{a}^{*, b} f\right)(x) \leq C\|b\|_{\theta}\left(\widetilde{M}_{r}\left(T_{a}^{*} f\right)(x)\right. & \\
& \left.+G_{4, r}^{N-\theta-n}(x)+\widetilde{M}_{r}(f)(x)\right) .
\end{aligned}
$$

This implies that

$$
\begin{gathered}
\left\|M_{\mathrm{loc}, 4}^{\sharp}\left(T_{a}^{*, b} f\right)\right\|_{L^{p}(w)} \\
\leq C\|b\|_{\theta}\left(\left\|\widetilde{M}_{p_{0}}\left(T_{a}^{*} f\right)\right\|_{L^{p}(w)}+\left\|G_{4, r}^{N-\theta-n} f\right\|_{L^{p}(w)}\right. \\
\left.+\left\|\widetilde{M}_{r}(f)\right\|_{L^{p}(w)}\right) .
\end{gathered}
$$

Since $\widetilde{M}_{r}, G_{4, r}^{N-\theta-n}$, and $T_{a}^{*}$ are bounded on $L^{p}(w)$ as long as $N>n+\theta+n / p+v / s$, we obtain the desired results.

This completes our proof.

\section{Acknowledgment}

The author would like to thank the referee for his/her useful comments and suggestions to improve the paper.

\section{References}

[1] J. Alvarez and J. Hounie, "Estimates for the kernel and continuity properties of pseudo-differential operators," Arkiv för matematik, vol. 28, no. 1, pp. 1-22, 1990.

[2] S. Chanillo and A. Torchinsky, "Sharp function and weighted $L^{p}$ estimates for a class of pseudo-differential operators," Arkiv för matematik, vol. 24, no. 1-2, pp. 1-25, 1985.
[3] L. Hörmander, "On the $L^{2}$ continuity of pseudopseudodifferential operators," Communications on Pure and Applied Mathematics, vol. 24, pp. 529-535, 1971.

[4] J. Hounie, "On the $L^{2}$ continuity of pseudopseudodifferential operators," Communications on Pure and Applied Mathematics, vol. 11, pp. 765-778, 1986.

[5] N. Michalowski, D. J. Rule, and W. Staubach, "Weighted norm inequalities for pseudo-pseudodifferential operators defined by amplitudes," Journal of Functional Analysis, vol. 258, no. 12, pp. 4183-4209, 2010.

[6] S. I. Nishigaki, "Weighted norm inequalities for certain pseudodifferential operators," Tokyo Journal of Mathematics, vol. 7, pp. 129-140, 1984.

[7] K. Yabuta, "Weighted norm inequalities for pseudodifferential operators," Osaka Journal of Mathematics, vol. 23, pp. 703-723, 1986.

[8] N. Miller, "Weighted Sobolev spaces and pseudodifferential operators with smooth symbols," Transactions of the American Mathematical Society, vol. 269, pp. 91-109, 1982.

[9] L. Tang, "Weighted norm inequalities for pseudo-differential operators with smooth symbols and their commutators," Journal of Functional Analysis, vol. 262, pp. 1603-1629, 2012.

[10] E. M. Stein, Harmonic Analysis: Real Variable Methods, Orthogonality and Oscillatory Integrals, Princeton University, Princeton, NJ, USA, 1993.

[11] C. E. Kenig and W. Staubach, " $\psi$-pseudodifferential operators and estimates for maximal oscillatory integrals," Studia Mathematica, vol. 183, no. 3, pp. 249-268, 2007.

[12] B. Bongioanni, E. Harboure, and O. Salinas, "Classes of weights related to Schrödinger operators," Journal of Mathematical Analysis and Applications, vol. 373, no. 2, pp. 563-579, 2011.

[13] B. Bongioanni, E. Haboure, and O. Salinas, "Commutators of Riezs transforms related to Schrödinger operators," Journal of Fourier Analysis and Applications, vol. 17, pp. 115-134, 2011.

[14] J. Dziubański and J. Zienkiewicz, "Hardy space $H^{1}$ associated to Schrödinger operator with potential satisfying reverse holder inequality," Revista Matematica Iberoamericana, vol. 15, no. 2, pp. 279-296, 1999.

[15] G. Pradolini and O. Salinas, "Commutators of singular integrals on spaces of homogeneous type," Czechoslovak Mathematical Journal, vol. 57, no. 1, pp. 75-93, 2007. 


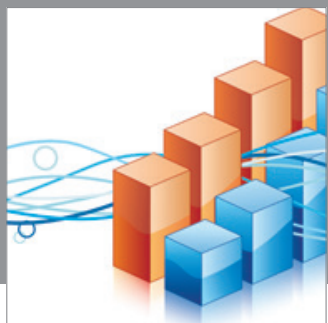

Advances in

Operations Research

mansans

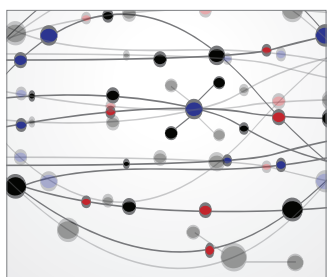

The Scientific World Journal
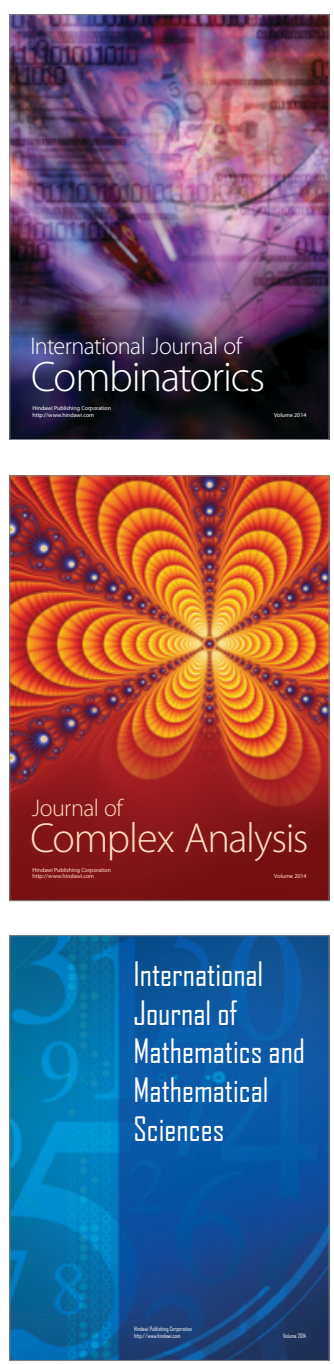
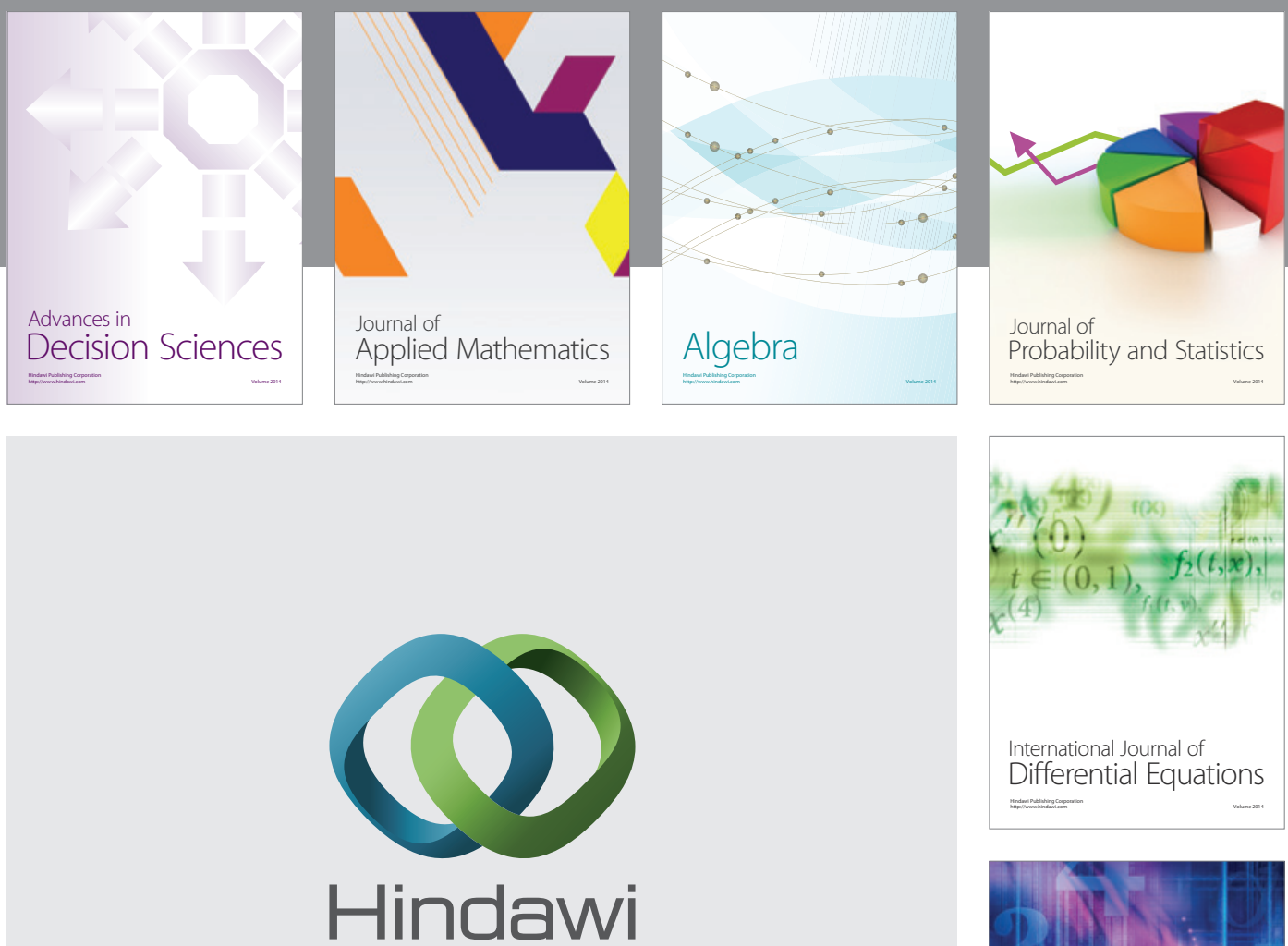

Submit your manuscripts at http://www.hindawi.com
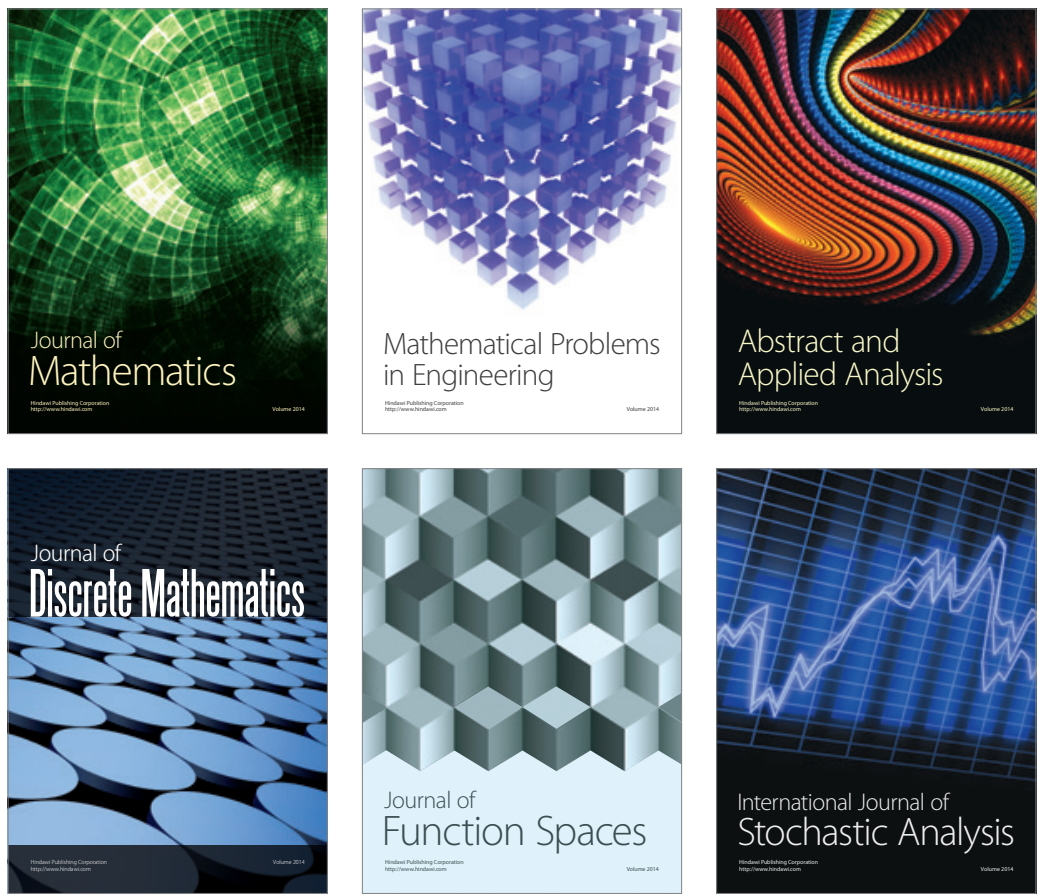

Journal of

Function Spaces

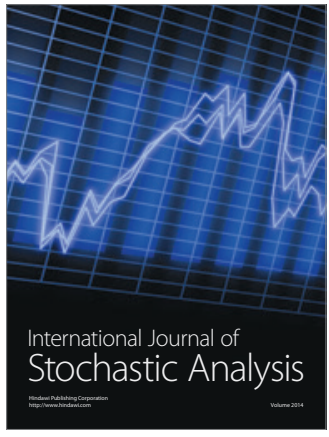

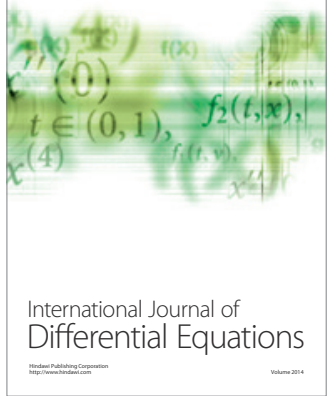
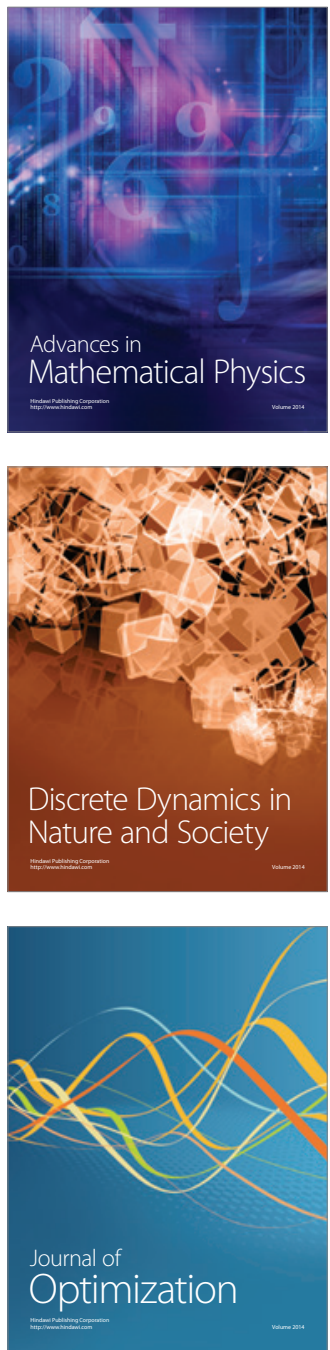